As per ESC: E-EPA $2 \times 2$ g per day should be considered in combination with a statin for patients with:

- Persistently high TGs (1.5-5.6 mmol/L)

- Treatment with a statin

Results 398 patients completed cardiac rehab during this study and were included in our database. Of these 275 (69\%) had a 6 month TG and LDL recorded and were included. All patients in our cohort had been on a stable dose of statin for at least 4 weeks.

Analysis as per initial REDUCE IT protocol: 14/275 patients $(5 \%)$ were excluded as they were less than 45 years old. 63 patients (23\%) had a TG level of $1.5-5.6$ and 42 of these had an LDL-C level of 1.06-2.59 mmol/L (15.3\%). This led to an overall eligibility of $15.3 \%$ for E-EPA.

Analysis as per amended REDUCE-IT protocol: 14/275 patients $(5 \%)$ were excluded as they were less than 45 years old. 30 patients had a TG level eligible as per the amended REDUCE-IT protocol (10.9\%) and 20 of these patients had an LDL-C level of $1.06-2.59 \mathrm{mmol} / \mathrm{L}$ (7.3\%) leading to an overall eligibility of $7.3 \%$ for E-EPA.

Analysis as per the ESC/EAS guidelines: Notably the ESC guidelines do not specify an LDL level or age required for EEPA to be considered. 64 patients had a TG level of 1.5-5.6 mmol/L despite statin therapy. As such, 64/275 patients (23.3\%) of our cohort would be eligible for E-EPA.

Conclusions E-EPA is a dominant cost-effective strategy to reduce $\mathrm{CV}$ risk in patients with elevated TG levels despite statin therapy.

Nearly one quarter (23.3\%) of patients in our cohort would be suitable for E-EPA treatment in order to further reduce their $\mathrm{CV}$ risk.

Rehab services should develop screening strategies to identify and treat patients eligible for E-EPA therapy.

\section{INFLUENZA VACCINE UPTAKE IN A CARDIOLOGY OUTPATIENT DEPARTMENT SETTING; A MISSED CARDIOVASCULAR DISEASE PREVENTION OPPORTUNITY}

N Connolly. Galway University Hospital, Galway, Ireland

\subsection{6/heartjnI-2020-ICS.55}

Aim Management of chronic illness is a cornerstone of care in the secondary care setting. Cardiovascular patients may have multiple indications for influenza vaccination and protection from Flu may improve both mortality and morbidity. Studies have shown a direct link between inflammation, respiratory illness and acute myocardial infarction and there is existing evidence that influenza vaccination reduces acute MI rates in both primary and secondary prevention. The aim of this audit was to evaluate if patients attending a cardiology outpatient department, with an indication for influenza vaccination, as per current guidelines, are adequately vaccinated and if an opportunity lies there in to target this patient cohort and improve outcomes.

Method Patients attending two large university teaching hospital outpatient departments were asked to fill out a survey, including simple personal medical history details, seeking to identify; patients with an indication for vaccination, patients that had been offered vaccination and reasons for refusal if they had been offered but declined. Unvaccinated patients were asked if would they avail of vaccination if it were available on the day at the clinic. The results were collated and reviewed.

Results 142 respondents in total; average age 66.2 yrs (STD 14.2), $64.7 \%$ male, $79.6 \%>50$ yrs, $28.9 \%$ attended a Heart Failure Unit, $81.7 \%$ overall had an indication for vaccination other than age alone and $90.8 \%$ had an indication when age $>50$ yrs was also included. Of the patients with an indication for vaccination, vaccination rates were; $62.9 \%$ males, $60.0 \%$ females, 73.2\% Heart Failure clinic attendees, 56.8\% General Cardiology attendees $(p=0.075$ for difference in vaccination rates among clinic types). Of the patients with an indication for vaccination that had not been vaccinated thus far, $48.9 \%$ would have received the vaccine on the day if offered. This would have improved the overall vaccination rate amongst patients with an indication for vaccination from $62.0 \%$ to $80.6 \%(p=0.015)$. Of the patients who would not opt to receive the vaccine if offered, $23 \%$ believe it doesn't work, $23 \%$ believe they would contract influenza from it and $38.5 \%$ have plans to receive the vaccine elsewhere.

\section{OPTIMISING LIPID TREATMENT FOLLOWING MYOCARDIAL INFARCTION}

${ }^{1} \mathrm{C}$ Cartmill, ${ }^{1}$ I Menown, ${ }^{2} \mathrm{G}$ Klabbers. ${ }^{1}$ Craigavon Cardiac Centre, Craigavon, UK; ${ }^{2}$ Faculty of Health, Medicine and Life Sciences, Maastricht University, Netherlands

\subsection{6/heartjnl-2020-ICS.56}

Introduction European Society of Cardiology (ESC) guidelines recommend intensive control of LDL cholesterol (LDL-C) following myocardial infarction (MI) to improve outcome. Early assessment of lipids post MI is confounded by acute phase response requiring re-testing to guide need for up-titration \pm additional treatment.

Method We studied patients admitted with MI across a healthcare region including 2 acute receiving hospitals over two years(2017-2018). Diagnosis, cardiovascular (CV) risk factors, CV history $(\mathrm{Hx})$, lipid treatment before admission, lipid profile on admission, lipid treatment on discharge, lipid profiles at first and second follow up, changes to lipid treatment and readmission were recorded. Chi-squared was used to assess relationships between variables.

Results Of 638 acute MI admissions, 227(35.6\%) had ST-elevation MI, 464(72.7\%) were male, 174(27.3\%) female. Baseline CV risk factors included diabetes $137(22.3 \%)$, family $\mathrm{Hx}$ 291(52.8\%), smoking [current 188(30.9\%); ex 164(26.9\%)], CV Hx 359(58.1\%). Lipid profile was tested on admission in $431(67.7 \%)$ subjects. For those already on lipid treatment, mean LDL-C was $2.22 \mathrm{mmol} / \mathrm{l}$; for those not, mean was 2.91 mmol/l. Almost all (98.3\%) were prescribed lipid lowering therapy prior to discharge (Atorvastatin 92.0\%, Simvastatin 2.1\%, Rosuvastatin 5.1\%, Pravastatin 0.3\%, Ezetimibe 0.5\%). A high intensity statin was used in $94.4 \%$ of the sample. Mean time to first follow-up lipid profile was 5.65 months. Follow up profiles were available in $85.6 \%$, in whom mean LDL-C was $1.67 \mathrm{mmol} / \mathrm{l}$. At first follow up 349(54.7\%) met the 2018 ESC target $<1.8 \mathrm{mmol} / \mathrm{l}$. For those not at target, 62 $(32.8 \%)$ received no further lipid testing and 13(6.9\%) had therapy increased. At final lipid test, $62.7 \%$ achieved LDL-C $<1.8 \mathrm{mmol} / \mathrm{l}$. Males $(\mathrm{p} \leq 0.1)$ and diabetics $(\mathrm{p}=0.01)$ were more likely to achieve target. Females were more likely to receive a lower dose of Atorvastatin $(p=0.004)$. There was no significant relationship between diabetes and discharge on 
higher intensity therapy $(\mathrm{p}>0.05)$. Only 207 (37.9\%) achieved the 2019 ESC target LDL-C $<1.4 \mathrm{mmol} / \mathrm{L}$. 36(6.6\%) retained high LDL-C $>3 \mathrm{mmol} / \mathrm{L}$ and $23(4.3 \%)$, very high LDL-C $>3.5 \mathrm{mmol} / \mathrm{L}$. Post discharge, 105(16.7\%) received changes to lipid therapy; 32(5\%) increased, 73(11.4\%) decreased. A cardiac-related readmission occurred in 140 (21.9\%).

Conclusion In this large sample, baseline lipid profiles were available in only $2 / 3$ patients and although follow up samples were available in $85.6 \%$, the mean time for first follow up was double the recommended 3 months losing early opportunity for up-titration. Such follow up is of high clinical importance as, despite high use of intensive statin therapy, over $1 / 3$ patients failed to achieve LDL-C $<1.8 \mathrm{mmol} / \mathrm{L}$. Fewer female patients were discharged on $80 \mathrm{mg}$ dose and were less likely to achieve target highlighting the importance of follow up in this group. At follow up only $<5 \%$ met current NICE guidelines for use of PCSK9i yet $2 / 3$ failed to achieve the current LDL-C target $<1.4 \mathrm{mmol} / \mathrm{L}$, suggesting a need for greater use Ezetimibe \pm PCSK9i therapy.

\section{PERCUTANEOUS TRANSFEMORAL TREATMENT OF LVOT PSEUDOANEURYSMS FOLLOWING BIOPROSTHETIC AVR}

PF Brennan, O Nzewi, MS Spence, C Owens. Royal Victoria Hospital, Belfast, UK

\subsection{6/heartjnl-2020-ICS.57}

Introduction Surgical aortic valve replacement (AVR) is the intervention of choice for many patients with aortic valve disease. Left ventricular outflow tract (LVOT) pseudo-aneurysm is a rare complication after AVR but, importantly, can present incidentally in the absence of specific symptoms. With progression the pseudo-aneurysm can be complicated by potentially fatal consequences including tract thrombosis, fistula formation, pseudo-aneurysmal rupture and compression of surrounding structures e.g. the coronary arteries.

Methods We report two cases of LVOT pseudo-aneurysm following SAVR, the various diagnostic/treatment-planning imaging modalities used, and the subsequent successful percutaneous interventions applied.

Results The first patient is a 69 -year-old female who underwent elective SAVR with a $25 \mathrm{~mm}$ Trifecta prosthesis. She had a history of bicuspid aortic valve disease (figure 1a). After an uncomplicated surgical course, she attended for a routine outpatient transthoracic echocardiogram (TTE). At this stage she was asymptomatic. An anechoic space was identified $15 \mathrm{~mm}$ adjacent to the AVR, in the non-coronary cusp position. There was evidence of bidirectional flow within the space. She was admitted to hospital and a TEE was performed which confirmed a $15 \mathrm{~mm} \times 27 \mathrm{~mm}$ echo free space adjacent to the aortic root (figure $1 \mathrm{~b}$ ) and colour doppler flow mapping demonstrated continuity of this space with the LVOT (figure 1c). Cardiac CT confirmed the presence of an LVOT pseudoaneurysm (figure 1d). The second patient is 76-year-old female who underwent elective SAVR with a $19 \mathrm{~mm}$ Trifecta prosthesis for severe trileaflet aortic stenosis. She was readmitted, 2-months later, with chest pain and dyspnoea. Due to concerns regarding an acute aortic syndrome a CT aorta was performed which suggested an LVOT pseudo-aneurysm arising in the immediate infra-annular vicinity. Subsequent TEE and cardiac-gated CT confirmed a $4 \times 9 \times 41 \mathrm{~mm}$ complex pseudo-aneurysm. Both patients were discussed with the heart team and the consensus was re-operation carried significant risk and to proceed to transfemoral percutaneous closure. The pseudo-aneurysms were easily visible during fluoroscopic angiography in the cathlab (figure 2a) and were both cannulated with guide extension catheters (figure 2b). Under general anaesthesia with TEE guidance both pseudoaneurysms were successfully using a single AVP2 $10 \mathrm{~mm}$ closure device (figure 2c). Repeat CT at 3 months revealed successful closure and placement of the devices (figure 2d) for each patient. At most recent followup they both remain asymptomatic, admission-free and have normal bioprosthetic valve function. Table 1 is a summary of both cases.

Conclusion LVOT pseudoaneurysms, a rare complication of SAVR, can be successfully treated percutaneously. In an era of

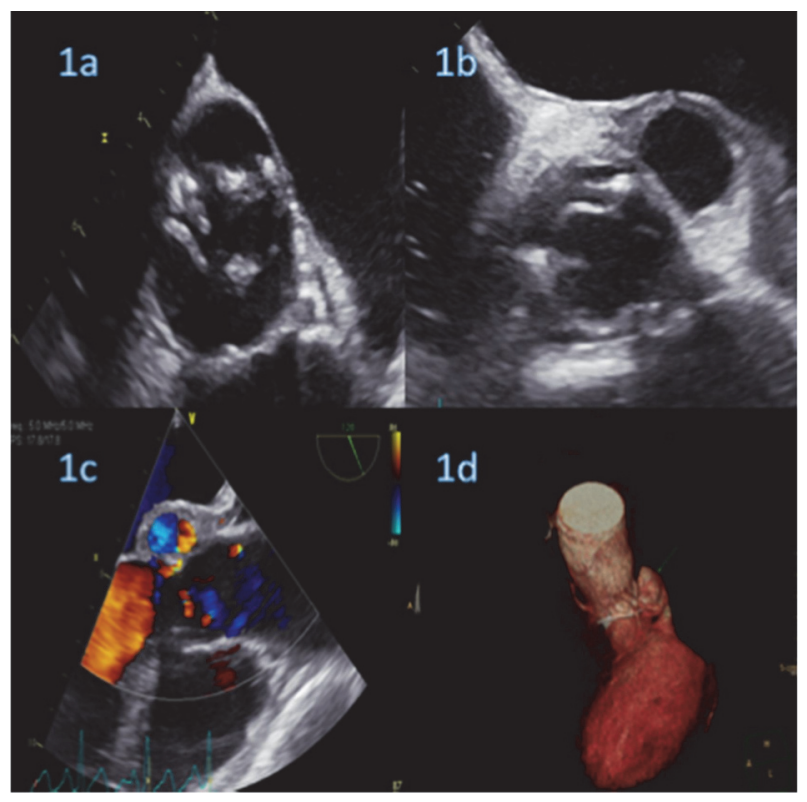

Abstract 57 Figure 1 Patient 1

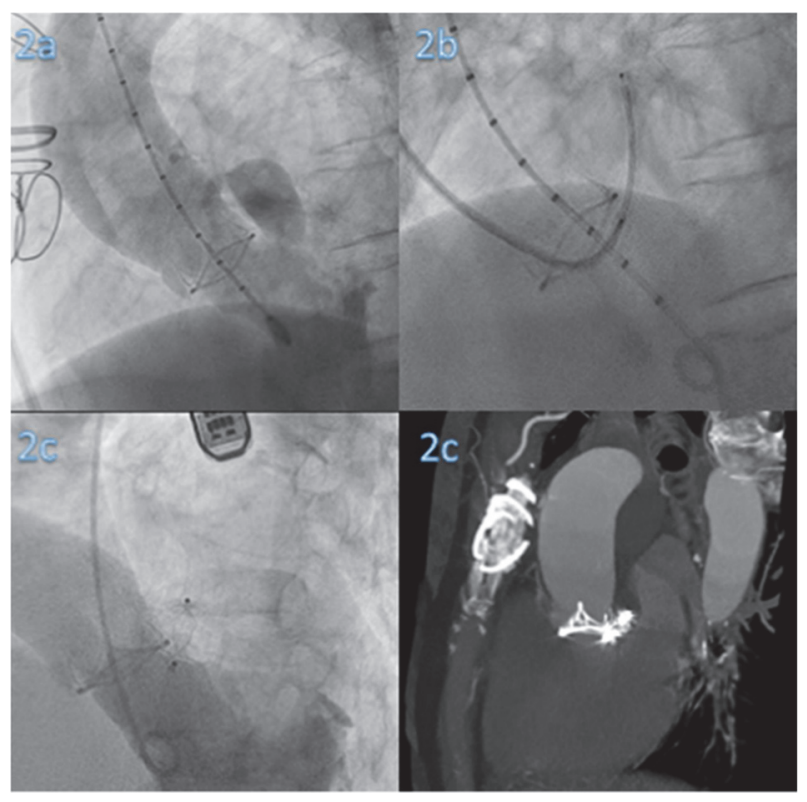

Abstract 57 Figure 2 Patient 2 